\title{
Hierarchical Fuzzy Systems Integrated with Particle Swarm Optimization for Daily Reference Evapotranspiration Prediction: A Novel Approach
}

Dilip Kumar Roy ( $\nabla$ dilip.roy@my.jcu.edu.au )

Bangladesh Agricultural Research Institute https://orcid.org/0000-0002-7685-0445

Kowshik Kumar Saha

Technical University of Berlin: Technische Universitat Berlin

Mohammad Kamruzzaman

Bangladesh Rice Research Institute

Sujit Kumar Biswas

Bangladesh Agricultural Research Institute

Mohammad Anower Hossain

Bangladesh Agricultural Research Institute

\section{Research Article}

Keywords: Reference evapotranspiration, Hierarchical fuzzy systems, Fuzzy inference system, Regression tree, M5 model tree, Shannon's entropy

Posted Date: July 21 st, 2021

DOI: https://doi.org/10.21203/rs.3.rs-617105/v1

License: (9) (1) This work is licensed under a Creative Commons Attribution 4.0 International License.

Read Full License

Version of Record: A version of this preprint was published at Water Resources Management on November 3rd, 2021. See the published version at https://doi.org/10.1007/s11269-021-03009-9. 


\section{Hierarchical fuzzy systems integrated with particle swarm optimization for daily reference evapotranspiration prediction: A novel approach}

Dilip Kumar Roy ${ }^{a^{*}}$, Kowshik Kumar Saha ${ }^{\mathrm{b}}$, Mohammad Kamruzzaman ${ }^{\mathrm{c}}$, Sujit Kumar Biswas ${ }^{\mathrm{a}}$ and Mohammad Anower Hossain ${ }^{a}$

arrigation and Water Management Division, Bangladesh Agricultural Research Institute, Gazipur - 1701, Bangladesh

${ }^{b}$ Department of Agromechatronics, Technical University of Berlin, 10623 Berlin, Germany

${ }^{c}$ Farm Machinery and Postharvest Technology Division, Bangladesh Rice Research Institute, Gazipur-1701, Bangladesh

*Corresponding author email: dilip.roy@my.jcu.edu.au

1

2

\section{Abstract}

Reference evapotranspiration $\left(\mathrm{ET}_{0}\right)$ is a crucial element for deriving a meaningful scheduling of irrigation for major crops. Thus, precise projection of future $\mathrm{ET}_{0}$ is essential for better management of scarce water resources in many parts of the globe. This study evaluates the potential of a Hierarchical Fuzzy System (HFS) optimized by Particle Swarm Optimization (PSO) algorithm (PSO-HFS) to predict daily $\mathrm{ET}_{0}$. The meteorological variables and estimated $\mathrm{ET}_{0}$ were employed as inputs and outputs, respectively, for the PSO-HFS model. The FAO 56 PM method to ET 0 computation was implemented to obtain $\mathrm{ET}_{0}$ values using the climatic variables obtained from two weather stations located in Gazipur Sadar and Ishurdi, Bangladesh. Prediction accuracy of PSOHFS was compared with that of a FIS, M5 Model Tree, and a Regression Tree (RT) model. Several statistical performance evaluation indices were used to evaluate the performances of the PSO-HFS, FIS, M5 Model Tree, and RT in estimating daily $\mathrm{ET}_{0}$. Ranking of the models was performed using the concept of Shannon's Entropy that accounts for a set of performance evaluation indices. Results revealed that the PSO-HFS model performed better than the tree-based models. 
15 Generalization capabilities of the preposed models were evaluated using the dataset from a test

16 station (Ishurdi station). Results revealed that the models performed equally well with the unseen

17 test dataset, and that the PSO-HFS model provided superior performance over other tree based

18 models. The overall results imply that PSO-HFS model could effectively be utilized to model $\mathrm{ET}_{0}$

19 values quite efficiently and accurately.

\section{Keywords:}

21 Reference evapotranspiration, Hierarchical fuzzy systems, Fuzzy inference system, Regression

22 tree, M5 model tree, Shannon's entropy

\section{1. Introduction}

24 Irrigating crops to enhance agricultural productivity essentially require sufficiently large volumes 25 of fresh water. Water-saving through carefully managed irrigation practices can be achieved 26 through precise quantification of evapotranspiration (ET), which is used to develop correct

27 irrigation scheduling, determine hydrologic water balances, simulate crop yields, and allocate 28 water resources (Kisi 2016). Being an essential component of water balance, ET plays a vital 29 function in controlling interactions among atmosphere, soil, and the vegetation (Liu et al. 2013). 30 The measurement of ET may be performed through experimental methods including Bowen ratio 31 energy balance method, eddy-covariance systems, and lysimeter techniques (direct methods) 32 (Martí et al. 2015). Alternatively, ET can be obtained by calculating potential or reference 33 evapotranspiration $\left(\mathrm{ET}_{0}\right)$ using climatological variables. This indirect method has become popular 34 in many parts of the world where direct measurements are not available or affordable due to 35 complexity or costliness (Allen et al. 1998). As a universal approach of $\mathrm{ET}_{0}$ estimation, the Penman 36 Monteith (FAO 56 PM) method has been recognized as the widespread reference method that can 37 be employed in arears with varying ecological and climatic circumstances with no requirement of 
regional adjustments (Allen et al. 1998). FAO 56 PM equation can be utilized to estimate $\mathrm{ET}_{0}$, which together with crop coefficient value provides an estimate of ET for a particular crop.

In recent years, artificial intelligence or machine learning based models have effectively been employed to model $\mathrm{ET}_{0}$ in different hydrogeologic conditions. These models can map the complex and nonlinear relations between the input and output data quite effectively and accurately. Various models have been used in $\mathrm{ET}_{0}$ modeling; among them, Artificial Neural Network (ANN) (Gocić and Arab Amiri 2021) models were the first implementation of machine learning tools to estimate $\mathrm{ET}_{0}$. Other recent implementation of machine learning tools in $\mathrm{ET}_{0}$ modelling includes the use of Adaptive Neuro Fuzzy Inference System (ANFIS) (Petković et al. 2020; Roy et al. 2020), Gaussian Process Regression (GPR) (Karbasi 2018), Gene-Expression Programming (GEP) (Wang et al. 2019), M5 Model Tree (M5Tree) (Kisi 2016), Multivariate Adaptive Regression Splines (MARS) (Kisi 2016), Random Forest (RF) (Wang et al. 2019; Ferreira and da Cunha 2020; Salam and Islam 2020), and Support Vector Machine (SVM) (Chia et al. 2020; Salam and Islam 2020). Generally, artificial intelligence based models have provided superior performances over the typical equations in estimating $\mathrm{ET}_{0}$, attaining relatively better performances with similar datasets (Reis et al. 2019). Among various machine learning algorithms, tree-based algorithms such as Random Forests, Regression Trees, and M5 Model Tree have recently been gained significant attention due to their simplicity, robustness, and capability to provide accurate predictions of $\mathrm{ET}_{0}$ (Chen et al. 2020). On the other hand, machine learning models derived from the theory fuzzy logic have recently been utilized as an effective prediction system in various water resources management issues (Kord and Asghari Moghaddam 2014). Although an ANFIS, a variant of Fuzzy Inference System (FIS) has been successfully applied in developing $\mathrm{ET}_{0}$ prediction models, its use is hindered by the computational burden arising from a large number of rule bases especially for problems with larger 
61 input variables. This happens because the number of rules in a fuzzy system escalates

62 exponentially with the quantity of variables inputted to the system. Larger rule bases make the

63 learning and fine-tuning of the rules and membership function parameters extremely challenging.

64 In addition, larger rule bases reduce the generalization capability of tuned fuzzy systems when

65 there exists insufficient training data. To overcome this issue, a FIS may be represented as a tree

66 of smaller interrelated and interconnected FIS objects known as Hierarchical Fuzzy Systems

67 (HFS), where the predictions from the lower-level FISs are utilized as predictors to the higher-

68 level FISs making the fuzzy tree-based HFS computationally more efficient than a single

69 monolithic FIS object.

70 HFS is an improved version of decision trees that provide reliable modelling using the concept of

71 fuzzy logic principle. Although applied quite successfully in various research domains (Zheng et

72 al. 2019), HFS models have been given extremely little attention in the fields of hydrological and

73 agricultural research. Few recent studies related to hydrology and water resources management

74 also focused on the use of fuzzy logic based decision trees. For instance, Sikorska-Senoner and

75 Seibert, (2020) employed a fuzzy logic based decision tree instead of a traditional trend analysis

76 for quantifying the magnitudes and frequencies in the time series of floods. Wei and Hsu, (2008)

77 performed a comparison between three types of decision trees: neural decision trees, conventional

78 decision trees, and fuzzy decision trees to derive operating rules for a reservoir operation system.

79 Their comparison results demonstrated the superiority of fuzzy decision trees over the other two

80 types of decision trees. Han et al., (2002) addressed uncertainty in real time flood forecasting using

81 fuzzy logic based fuzzy decision trees. They concluded that although fuzzy decision trees did not

82 perform as good as the ANN models for river flow modelling in the test case, the glass box nature

83 of fuzzy tree modelling could allow several valuable insights on the hydrological processes. As far 
84 as the recent literature is concerned, fuzzy tree models have not yet been used in hydrological and 85 agricultural research, especially in modelling $\mathrm{ET}_{0}$.

86 Considering the importance of reliable estimates of $\mathrm{ET}_{0}$, the purposes of this study were to: (1)

87 assess the potentiality of PSO tuned HFS model (PSO-HFS) to predict daily ET0; (2) weigh against

88 the prediction capability of the proposed PSO-HFS with that of two tree-based machine learning 89 algorithms including RT, and M5 Model Tree and a fuzzy logic-based model, FIS; (3) rank the 90 proposed models with respect to their prediction accuracies utilizing several performance 91 evaluation indices; and (4) evaluate the generalization capability of the proposed models outside 92 the training station using data from a test station. Accordong to the authors' understanding, this 93 study is the first effort an evolutionary algorithm-tuned fuzzy decision tree (PSO-HFS) is 94 employed to predict daily $\mathrm{ET}_{0}$.

\section{Materials and methods} was then compared with that of three machine learning algorithms: a fuzzy logic-based FIS model and two tree-based models. Comparison of prediction performances was evaluated using several statistical indices within the framework of Shannon's entropy that incorporated three benefits

101 (higher values indicate better model performance: Correlation Coefficient, Nash Sutcliffe Efficiency Coefficient, and Index of Agreement) and three cost indices (smaller values indicate better model performance: Root Mean Squared Error, Mean Absolute Error, and Median Absolute 104 Deviation) in the decision-making process. The proposed methodology was evaluated using the daily climatic data obtained from a weather station located in the Gazipur in Bangladesh. The 106 developed models were then validated using daily climatic data from Ishurdi meteorological 
107 station in Bangladesh. A brief description of methodology components is presented in the 108 subsequent subsections.

\subsection{Study area and the dataset}

110 Meteorological variables were acquired from two weather stations located in the Gazipur Sadar 111 Upazila of the Gazipur district and Ishurdi Upazilla of the Pabna district in Bangladesh. The 112 weather station in Gazipur is situated between $24.00^{\circ} \mathrm{N}$ latitude and $90.43^{\circ} \mathrm{S}$ longitude with an 113 altitude of $8.4 \mathrm{~m}$ above the mean sea level. Meteorological variables including solar radiation, 114 relative humidity, minimum and maximum temperatures, and wind speed were obtained for 15.5 115 years (from 01 January 2004 to 30 June 2019). Descriptive statistics of the meteorological 116 variables for the training station are given in Table 1. It is perceived from Table 1 that the 117 climatological variables demonstrated left (negative) skewness which indicates that the 118 distribution of data for all variables had an extended left tail than the right tail. Kurtosis, on the 119 other hand, had both positive and negative values indicating that the datasets had both "heavytailed" (positive values of kurtosis) and "light-tailed" (negative values of kurtosis) distributions.

122 The data for the test station were acquired from 01 June 2015 to 31 December 2020 (2021 daily 123 entries of meteorological variables and computed daily $\mathrm{ET}_{0}$ ). The performance evaluation indices 124 were calculated for the entire (2021 entries: from 01 June 2015 to 31 December 2020), first half 125 (1021 entries: from 01 June 2015 to 17 March 2018), and the second half (1020 entries: from 18 126 March 2018 to 31 December 2020) of the dataset for the test station. The selection of three sets of 127 data allows investigating a better generalization capability of the model. Descriptive statistics of 128 the meteorological variables of the test station are presented in Table 2. The locations of the 129 weather stations in the study areas are presented in Fig. 1. 
131 Meteorological variables obtained from the study areas across the period of study were utilized to 132 estimate daily $\mathrm{ET}_{0}$ by employing the FAO $56 \mathrm{PM}$ equation. These computed daily values of $\mathrm{ET}_{0}$ and the meteorological variables were used as outputs and inputs, respectively for the proposed HFS and other models. This indirect approach of $\mathrm{ET}_{0}$ estimation from meteorological variables has been widely accepted in circumstances when $\mathrm{ET}_{0}$ values are extremely hard to acquire directly (Allen et al. 1998). The FAO 56 PM equation is represented by:

$$
\mathrm{ET}_{0}=\frac{0.408 \Delta\left(R_{n}-G\right)+\gamma \frac{900}{T_{\text {mean }}+273} u_{2}\left(e_{s}-e_{a}\right)}{\Delta+\gamma\left(1+0.34 u_{2}\right)}
$$

where, $\mathrm{ET}_{0}$ represents reference evapotranspiration, $\mathrm{mm} \mathrm{d}^{-1} ; \mathrm{R}_{\mathrm{n}}$ is the net radiation at the crop surface, $M J \mathrm{~m}^{-2} \mathrm{~d}^{-1} ; \mathrm{G}$ is the heat flux density of soil, $\mathrm{MJ} \mathrm{m}^{-2} \mathrm{~d}^{-1} ; \Delta$ is the slope of the saturation vapor pressure curve, $\mathrm{kP}_{\mathrm{a}}{ }^{\circ} \mathrm{C}^{-1} ; \gamma$ is the psychometric constant, $\mathrm{kP}_{\mathrm{a}}{ }^{\circ} \mathrm{C}^{-1} ; \mathrm{e}_{\mathrm{s}}$ is the saturation vapor pressure, $\mathrm{kP}_{\mathrm{a}} ; \mathrm{e}_{\mathrm{a}}$ is the actual vapor pressure, $\mathrm{kP}_{\mathrm{a}}$; $\mathrm{u}_{2}$ is the wind speed at a height of $2 \mathrm{~m}, \mathrm{~m} \mathrm{~s}^{-1}$; and $\mathrm{T}_{\text {mean }}$ is the mean air temperature at $2.0 \mathrm{~m}$ height, ${ }^{\circ} \mathrm{C}$.

[Fig. 1]

For the training station (Gazipur Sadar), computed $\mathrm{ET}_{0}$ values ranged between 0.92 and 8.02 $\mathrm{mm} \mathrm{d}^{-1}$ with the mean and standard deviation values of 3.80 and $1.32 \mathrm{~mm} \mathrm{~d}^{-1}$, respectively. The distribution of $\mathrm{ET}_{0}$ time-series had an extended right tail compared to the left tail as indicated by a positive skewness value of 0.30 . The negative kurtosis value of -0.67 indicates a "light-tailed" distribution for the computed $\mathrm{ET}_{0}$ values of the train ststion. On the other hand, the mean, standard deviation, skewness, and kurtosis values of the computed $\mathrm{ET}_{0}$ for the entire dataset of the test station were $3.67 \mathrm{~mm} \mathrm{~d}^{-1}, 1.24 \mathrm{~mm} \mathrm{~d}^{-1}, 0.28$, and -0.62 , respectively. For the first half of the dataset, the values were $3.57 \mathrm{~mm} \mathrm{~d}^{-1}, 1.25 \mathrm{~mm} \mathrm{~d}^{-1}, 0.35$, and -0.62 , respectively. The second half of the 
151 dataset comprised the following values of $\mathrm{ET}_{0}$ : mean $=3.76 \mathrm{~mm} \mathrm{~d}^{-1}$, standard deviation $=1.23 \mathrm{~mm}$

$152 \mathrm{~d}^{-1}$, skewness $=0.22$, kurtosis $=-0.59$.

154 Fuzzy Inference Systems (FIS) are regarded as one of the most effective tools for modelling 155 dynamic and nonlinear systems with single output and multiple inputs (Takagi and Sugeno 1985; 156 Sugeno and Yasukawa 1993). However, the computational efficiency during the FIS training 157 largely relies on the quantity of inputs to the FIS system and the quantity of rule sets, which 158 generally increase exponentially as the number of input variables increases. A significant amount 159 of rule sets not only reduces the computational efficiency but also creates difficulty in the tuning 160 process of the rule base and membership function parameters. Moreover, an increased number of 161 rule bases reduces the generalization capability of tuned FISs especially in situations where the 162 amounts of training data are scarce as can be seen in many practical applications. As a solution to 163 these problems an HFS consisting of smaller interconnected FIS objects can be implemented 164 instead of a single massive FIS object with many input variables. In an HFS, the fuzzy inference 165 systems are organized in 'hierarchical tree structures' in which the predictions from the lower166 level FISs are employed as predictors to the higher-level FISs. With a similar number of input 167 variables, an HFS usually requires fewer computation efforts compared to a single FIS. Fuzzy tree 168 structures, based on which an HFS is constructed, can be of three major types for many practical 169 applications: (a) incremental, (b) aggregated, and (c) cascaded or combined that combines both 170 incremental and aggregated structures (Siddique and Adeli 2013). As cascaded tree structures are 171 better suited for applications with both uncorrelated and correlated input variables, a cascaded or 172 combined fuzzy tree structure is utilized in this research for constructing an HFS. 
173 The first step of developing an HFS involves the creation of several FIS objects using the available

174 input variables ranked based on their correlations with the output variable $\left(\mathrm{ET}_{0}\right)$. Both positively

175 and negatively correlated input attributes were used to incorporate both the positive and negative

176 impacts of input attributes on the output $\left(\mathrm{ET}_{0}\right)$ for prediction. Next, in the second step, the input

177 attributes were paired concerning their ranks to create individual FIS object as follows:

178

179

180

181

182

183

184

185

186

187

188

189

190

191

192

193

194

195

- fis1: Maximum Temperature and Relative Humidity

- fis2: Wind Speed and Sunshine Duration

- fis3: Minimum Temperature and Sunshine Duration

[Fig. 2]

Then, the HFS as shown in Fig. 2, was constructed using the principle of FIS tree structure (Mathworks 2021). The constructed HFS had five two-input and one-output FIS objects (fis1, fis2, fis3, fis4, and fis5 in Fig. 2) of which the first three FISs (fis1, fis2 and fis3) received the ranked input attributes directly and produced intermediate predictions of $\mathrm{ET}_{0}$. The intermediate $\mathrm{ET}_{0}$ values were integrated utilizing the remaining two FISs (fis4 and fis5).

\subsection{Other prediction models}

2.3.1. Fuzzy inference system (FIS)

The underlying principles of FIS are derived from fuzzy set theory that has received considerable attention in recent years. FISs have successfully been applied as a reliable computing framework in various research areas (Jang et al. 1997). They have been regarded as effective prediction tools for modelling nonlinear processes due to their capability of accurately mapping of the relationships (usually nonlinear) between input and output variables (Takagi and Sugeno 1985; Sugeno and Yasukawa 1993). A Sugeno Sugeno-type FIS (Sugeno, 1985), also known as a Takagi-SugenoKang FIS, is particularly better fitted for nonlinear system modelling. A Sugeno FIS builds input- 
output relationships through interpolating the outputs from multiple linear models. The basic structure of a Sugeno FIS consists of a rule base, a database, and a reasoning mechanism. The working principle of a basic FIS with three inputs, one output, and four rules is illustrated in a block diagram as shown in Fig. 3.

[Fig. 3]

Rule bases of an FIS consist of fuzzy if-then rules, the database determines the types and numbers of MFs utilized in fuzzy rules, and finally, the reasoning mechanism accomplishes the fuzzy inference process (Jang et al. 1997). Several fuzzy if-then rules are utilized in a fuzzy inference process for producing a nonlinear mapping of input and output variables. A fuzzy rule consists of two parts: (a) antecedent part of any rule specifies a fuzzy region within the input variable space, and (b) consequent part specifies a fuzzy region within the output variable space. A Sugeno-type FIS introduced in 1985 (Sugeno 1985) was developed and utilized in this effort. The input and output MFs of the utilized Sugeno FIS were Gaussian and linear, respectively.

\subsubsection{M5 model tree}

The development of M5 model trees is based on the principles of the M5 method (Quinlan 1992). This method builds single trees corresponding to a method called 'M5', which makes use of the 'Standard Deviation Reduction' criterion. Model trees (MT) are combinations of traditional regression trees which possess linear regression functions at the leaf nodes. Pruning and smoothing operators determine the contents of a leaf node for a MT, which is nothing but a regression tree without 'pruning' and 'smoothing' operations. MTs (Quinlan 1992) are machine learning algorithms that have demonstrated their predictive capabilities in various research domains (Bhattacharya and Solomatine 2005). MTs are 'inverted trees', i.e., root nodes are situated at the upper portion of the tree whereas the bottom portion of the tree contains the leaves. 
219 MTs and regression trees are the variants of Decision Trees (DT) that have been established for

220 solving regression tasks (Quinlan 1992). However, MTs differ from regression trees: while MTs

221 generate linear models at their leaves, the regression trees yield a constant value at their leaves.

222 The linear models developed at the leaves are used to contain input-output relationships, which are

223 then utilized to predict outputs for a given set of data. MTs are more efficient than regression trees

224 in handling large datasets and producing more accurate predictions. M5 MT utilizes 'divide-and-

225 conquer' method that allows dividing the entire data space into smaller data sub-spaces

226 (Bhattacharya and Solomatine 2005). In this approach, the input parameter space is narrowed down

227 to several subspaces each of which represents a linear regression model. This unique data splitting

228 procedure enables M5 MT to produce a hierarchial model tree that contains splitting rules in its

229 non-terminal nodes and has expert models in its leaves.

230 A MATLAB toolbox “M5PrimeLab” (Jekabsons 2016) was employed to built M5 model trees for

231 predicting daily reference $\mathrm{ET}_{0}$ values with various climatic variables as inputs and $\mathrm{ET}_{0}$ values as

232 outputs.

\subsubsection{Regression tree}

234 Regression trees (RT) are decision trees that build simple, flexible, and easily interpretable models 235 developed using input-output training patterns. RTs are associated with the principle of 236 'Classification and Regression Tree (CART)' algorithm (Breiman et al. 1984; Krzywinski and 237 Altman 2017). The CART algorithm follows three major stepwise procedures in building models:

238 (a) building a complex tree, (b) pruning, and (c) selecting an optimal subtree. In the first step, a 239 complex full tree with several terminal nodes is built using a binary split procedure. The complex 240 tree built in the first step is pruned in the second step to prevent or at least reduce model overfitting.

241 In the third step, an optimal subtree is selected by the CART algorithm to ensure the quality of 
242 prediction for new samples. The developed RTs deliver a predicted response through the following 243 decisions from the beginning node (root node) to the leaf node within the tree. The leaf node of an

244 RT contains the responses or outputs. The RT-based $\mathrm{ET}_{0}$ prediction models were developed in the 245 MATLAB environment.

247 The meteorological variables and the estimated $\mathrm{ET}_{0}$ formed the input-output datasets for the 248 proposed $\mathrm{ET}_{0}$ prediction models. The dataset of the training station contains 5660 daily entries 249 (from 01 January 2004 to 30 June 2019) of meteorological variables and estimated ET values. 250 The entire dataset of 5660 entries was separated into training, validation, and test sets: first $80 \%$ 251 of the total data (4528 entries: from 01 January 2004 to 24 May 2016) was used to train and validate 252 the proposed models whereas the last 20\% (1132 entries: from 25 May 2016 to 30 June 2019) was 253 used to test the developed models. The first $80 \%$ of the sequential data were randomized to 254 minimize the effect of trends during the model training and validation process. The randomized 255 data were then split into two equal-sized datasets for training (first 40\%) and validation (remaining $25640 \%$ ) sets. It is noted that the test set (last $20 \%$ of the entire dataset) was kept in sequence as this 257 dataset was used to test the developed models for the actual nature of data. This technique of data 258 partitioning allows better performance evaluation for the developed models (Francone 2001). For 259 performance evaluation, numerous statistical indices were computed on the test dataset.

\section{Parameter tuning of HFS: Particle swarm optimization}

261 A parameter tuning approach was adopted to achieve optimum performance of the constructed

262 HFS. Both the rule bases and input-output Membership Functions (MF) were tuned in two 263 subsequent phases. In the first phase, learning of the rule bases was accomplished while input and 264 output MF parameters were kept constant. After learning the new rules through the first phase, the 
265 parameters of the input-output MF, as well as the learned rules, were tuned simultaneously in the

266 second phase. To achieve computational efficiency in the parameter tuning process, the tuned rule

267 base obtained from the first phase was used as the initial condition for the second phase. This

268 allows a fast parameter tuning process and quick convergence to global optima. Particle Swarm

269 Optimization (PSO) has recently gained popularity due to the possession of many advantageous

270 characteristics such as it has simple structure, robust maneuverability, and easy realization that

271 facilitates the training of various intelligent models. Therefore, this study utilized PSO in both

272 phases of parameter tuning to obtain optimal parameter values of the constructed HFS.

273 The PSO (Kennedy and Eberhart 1995), a swarm-based stochastic search algorithm, is encouraged

274 by communal and psychological principles. The PSO is associated with the principles of swarm

275 intellect, which feigns the communal behavior of predation commonly observed in fish schooling

276 or bird flocking. In PSO, every single particle is regarded as a 'feasible solution' over the entire

277 search space for a given optimization problem. On the other hand, the flight behavior of particles

278 is regarded as the search method for all individuals within a community. In PSO, the dynamic

279 update of the velocity of particles is determined by the past optimal location of the particle and the

280 population within a swarm. In PSO, the particle's objective function values are the corresponding

281 fitness values. These fitness values determine the optimum particle position. The fitness values are

282 also used to update the past most favorable location of the particles and the optimal location of the

283 swarm population. The control parameters of the PSO algorithm determine the convergence of

284 particle trajectories. Convergence of the PSO algorithm is attained through maintaining a memory

285 of standalone best fitness values of each particle, locating the global best particle, and bringing up

286 to date the location and velocity of all particles. If the convergence is not attained, the iterative 
287 process repeats till the optimization problem reaches to its optimal solution or the user defined 288 maximum quantity of iterations is attained.

\section{Implementation of the proposed HFS to model ETo}

290 In developing the proposed models, $\mathrm{ET}_{0}$ was considered as the target variable whereas the 291 meteorological variables were used as the inputs. The inputs to and outputs from the HFS can be 292 represented in the generalized form as:

$$
\text { Reference evapotranspiration, } E T_{0}=f(\text { meteorological variables })
$$

293 Determining the optimal parameter values which have a significant impact on the output variable

294 is the most important step in developing predictive models. Parameter tuning was performed using 295 a swarm-based optimization algorithm, PSO described earlier in the previous section (section 3:

296 Parameter tuning of HFS: Particle swarm optimization). To further improve the HFS model's 297 accuracy, all tunable parameters (rule bases and parameters of both input and output MFs) were 298 optimized using the PSO algorithm. The parameters of the PSO algorithm were selected upon 299 several trials concerning a tradeoff between prediction precision and computational efficiency.

300 Eventually, the input-output datasets used to construct the prediction models were split into train, 301 validation, and test sets. Additionally, FIS, M5 Model Tree, and RT-based ET 0 prediction models 302 were also developed solely for comparison purposes. Nonlinear mapping of inputs and outputs for 303 a typical $\mathrm{ET}_{0}$ prediction modelling approach can be schematically represented by Fig. 4.

\section{[Fig. 4]}

\section{Ranking of models: Shannon's entropy}

306 Shannon's entropy (Shannon 1948) was applied to assign weights to individual ET 0 prediction 307 models which were ranked according to the weights assigned to them. Shannon's entropy 
308 incorporates a set of benefit (the higher values indicate better model accuracy) and cost (the lower

309 values indicate better model accuracy) performance evaluation indices. The detailed calculation

310 steps of Shannon's entropy can be found in Roy et al. (2020), and are not repeated here.

\section{6. Performance evaluation criteria}

312 The proposed modelling approach was evaluated with several performance indicators as follows:

313 Root Mean Squared Error, RMSE:

$$
R M S E=\frac{\sqrt{\frac{1}{n} \sum_{i=1}^{n}\left(E T_{0_{i}}{ }^{A}-E T_{0_{i}}{ }^{P}\right)^{2}}}{\overline{E T_{0}{ }^{A}}}
$$

314 Normalized RMSE, NRMSE:

$$
N R M S E=\frac{R M S E}{\overline{E T_{0}{ }^{A}}}
$$

315 Accuracy:

$$
A c c=1-a b s\left(\operatorname{mean} \frac{E T_{0_{i}}{ }^{P}-E T_{0_{i}}{ }^{A}}{E T_{0_{i}}{ }^{A}}\right)
$$

316 Mean Bias Error (MBE):

$$
M B E=\frac{1}{n} \sum\left(E T_{0_{i}}^{P}-E T_{0_{i}}^{A}\right)
$$

317 Nash-Sutcliffe Efficiency Coefficient (NS):

$$
N S=1-\frac{\sum_{i=1}^{n}\left(E T_{0_{i}}{ }^{A}-E T_{0_{i}}{ }^{P}\right)^{2}}{\sum_{i=1}^{n}\left(E{T_{0}}_{i}^{A}-\overline{E T_{0}{ }^{A}}\right)^{2}}
$$

Willmott's Index of Agreement (IOA): 


$$
d=1-\frac{\sum_{i=1}^{n}\left(E T_{0_{i}}{ }^{A}-E T_{0_{i}}{ }^{P}\right)^{2}}{\sum_{i=1}^{n}\left(\left|E T_{0_{i}}{ }^{P}-\overline{E T_{0}{ }^{A}}\right|\left|E{T_{0}}^{A}-\overline{E T_{0}{ }^{A}}\right|\right)^{2}}
$$

319 Mean Absolute Error, MAE

$$
M A E=\text { Average }\left[\left|E T_{0_{i}}^{A}-E T_{0_{i}}^{P}\right|\right]
$$

320 Median Absolute Deviation, MAD

$$
\begin{gathered}
M A D\left(E T_{0_{i}}{ }_{i}^{A}, E T_{0_{i}}^{P}\right)=\operatorname{median}\left(\left|E T_{0_{1}}{ }^{A}-E T_{0_{1}}{ }^{P}\right|,\left|E T_{0_{2}}{ }^{A}-E T_{0_{2}}{ }^{P}\right|, \ldots,\left|E T_{0_{n}}{ }^{A}-E T_{0_{n}}{ }^{P}\right|\right) \\
\text { for } i=1,2, \ldots, n
\end{gathered}
$$

321 Correlation Coefficient (R):

$$
R=\frac{\sum_{i=1}^{n}\left(E T_{0_{i}}{ }^{A}-\overline{E T_{0}{ }^{A}}\right)\left(E T_{0_{i}}{ }^{A}-\overline{E T_{0}{ }^{P}}\right)}{\sqrt{\sum_{i=1}^{n}\left(E T_{0_{i}}{ }^{A}-\overline{E T_{0}{ }^{A}}\right)^{2}} \sqrt{\sum_{i=1}^{n}\left(E T_{0_{i}}{ }^{P}-\overline{E T_{0}{ }^{P}}\right)^{2}}}
$$

322 where, $E T_{0_{i}}{ }_{i}^{A}$ and $E T_{0_{i}}^{P}$ are the FAO $56 \mathrm{PM}$ estimated and model predicted $\mathrm{ET}_{0}$ values for the $i^{\text {th }}$ 323 data points of the dataset, respectively; $\overline{E T_{0}{ }^{A}}$ and $\overline{E T_{0}{ }^{P}}$ are the mean of the FAO 56 PM estimated 324 and model predicted $\mathrm{ET}_{0}$ values, respectively; $n$ is the number of data points in the dataset.

\section{7. Results and discussion}

326 The values of FAO 56 PM estimated $\mathrm{ET}_{0}$ were considered as the benchmark for evaluating the 327 performances of the proposed HFS, and other models (FIS, M5 Model Tree, and RT) developed 328 for comparison purposes. Ten statistical performance assessment indices were computed for both 329 the calibration (Training and Validation datasets) and testing (applied dataset) phases of model 330 building. Performance assessment indices were calculated on the FAO 56 PM estimated and model 331 predicted $\mathrm{ET}_{0}$ values. Performances of the developed models to the computed performance indices 332 for the calibration and testing phases are presented in the subsequent paragraphs. 
334 The training phase of model building is regarded as the most important step in the development of any prediction model. To prevent model over-or underfitting, training performance is compared with the validation performance using the validation data. Training and validation phases were

337 performed simultaneously, and several evaluation indices were calculated on the model predicted 338 and FAO 56 PM estimated $\mathrm{ET}_{0}$ values. Performances of the Proposed HFS and other tree-based 339 models during training and validation stages are shown in Table 3 . It is evidenced from Table 3 that all performance indices showed a reasonably good performance of the proposed HFS model during training and validation stages as evidenced by the negligible difference in values of the performance evaluation indices between these two phases.

\section{[Table 3]}

344 Although not as accurate as the proposed HFS model, the FIS-based model performed equally well 345 during the training and validation phases (Table 3). Training performances of RT and M5 Model 346 Tree were observed relatively better than their validation performances especially on the cost 347 indices (MAD, MAE, MAPRE, RMSE, and NRMSE) as shown in Table 3. However, their 348 performances on benefit indices (e.g., accuracy, IOA, NS, and R) were found to be almost similar 349 which indicates reasonably decent performance on the benefit indices. Overall, the training and 350 validation performances of the proposed HFS model were obtained superior to the other tree-based 351 models. Nevertheless, although performed differently on different performance indices as well as 352 on the training and validation phases, all models presented in Table 3 produced quite acceptable 353 results in the context of prediction modelling.

354 Although RT and M5 Model Tree suffered slightly from model overfitting as evidenced by the 355 results obtained from the cost indices, the models produced satisfactory results concerning the 
356 benefit indices. This is also acceptable because prediction models often show contradictory

357 performances, i.e., one model may be deemed suitable based on the RMSE criterion whereas the 358 other model may be a good performer based on the R criterion (Müller and Piché 2011; Roy and 359 Datta 2019, 2020). This conflicting nature of the prediction models necessitates the incorporation 360 of several performance evaluation indices instead of few indices within a framework of a decision 361 theory for judging the performance of any prediction model. A decision theory incorporating 362 Shannon's entropy based weighting system was applied to judge the performance and to rank the 363 developed models for the test performances of the trained and validated models (Subsection 7.3: 364 Ranking of the developed $\mathrm{ET}_{0}$ prediction models).

\subsection{Performance of the HFS during the testing phase}

366 The proposed HFS and other tree-based models were further tested using the test data which were 367 used neither to train nor to validate the models, i.e., the models were tested using data from outside 368 the training and validation datasets. The performances during the testing phase (with applied dataset) computed on the estimated FAO 56 PM and model-predicted (calibrated and validated) $\mathrm{ET}_{0}$ values using several performance evaluation indices are presented in Table 4.

372 As the results indicate, the models performed equally well when compared to the training and 373 validation phases. It is observed from Table 3 that although the performances were slightly poor 374 during the applied phase when compared to the calibration and validation phases, the model 375 performance during the applied phase was excellent in the context of prediction modelling. The 376 applied results produced accuracy $>0.97$, IOA $>0.99, \mathrm{NS}>0.99, \mathrm{R}>0.95, \mathrm{MAD}<0.2, \mathrm{MAE}<0.3$, 377 MAPRE $<8 \%$, and NRMSE $<0.1$ which indicate an excellent model performance. Models' 378 performance is deemed excellent when the NS statistic value is greater than 0.8 (Gupta et al. 1999) 
379 suggesting an efficient performance of the developed models. Moreover, the NRMSE (or scatter 380 index) values in the applied phase were 0.052 and 0.093 , respectively for the best (HFS) and the 381 worst (RT) model. These NRMSE values also illustrate the excellent performance of the developed 382 models based on the criteria set in Heinemann et al., (2012) and in Li et al., (2013). According to 383 them, model performance is said to be excellent when NRMSE value is lower than 0.1, good when 384 NRMSE value is between 0.1 and 0.2 , fair when NRMSE value is between 0.2 and 0.3 , poor when 385 NRMSE is greater than 0.3. In general, model performance for all the developed models was 386 satisfactory as indicated by the lower values of MAD, MAE, MAPRE, and NRMSE together with 387 higher values of accuracy, IOA, NS, and R.

388 The model performances were also evaluated based on scatter and regression plots presented in 389 Figs. 5 and 6. ET 0 estimates of the four modelling approaches with the benchmark FAO 56 PM 390 method during the test period were illustrated in Fig. 5. As can be observed, the HFS predictions 391 were nearer to the FAO 56 PM estimated ET $_{0}$ values than the FIS and RT models. On the other 392 hand, fluctuations of model predictions were remarkably close to each other in the case of the HFS 393 and M5 Model Tree. This confirms the calculated performance evaluation indices presented in 394 Table 3.

396 Comparison of the regression plots obtained from the model predictions and FAO 56 PM estimates 397 for the test dataset is illustrated in Fig. 6. It is apparent from the regression plots that the HFS had 398 fewer scattered predictions compared to the FIS and RT models, and was closely followed by the 399 M5 Model Tree. Regression plots confirmed the superior performance of the HFS model over the 400 FIS, RT, and M5 Model Tree. 
402 Prediction accuracies of the proposed HFS and three other tree-based models presented as box

403 plots of absolute errors (Fig. 7) reveal that the PSO tuned HFS model outperformed other

404 prediction models and that the accuracy of prediction for the M5 Model tree was better than FIS

405 and RT models. It is worth mentioning that the prediction accuracy of the M5 Model Tree was

406 equally well with the HFS model accuracy. However, the M5 Model Tree had more high

407 magnitude absolute error values when compared to the HFS model. Therefore, it is evidenced that

408 the HFS model is considered as the superior performer, among others.

[Fig. 7]

410 Prediction performances of the proposed HFS and other tree-based models were also evaluated

411 using spider plots, also termed as 'radar' (Rankin et al., 2008), which can assess the performance

412 of multifunctional systems. In a spider plot, relevant performance indices are chosen and assigned

413 to an axis on a multidimensional plot. Displaying relevant data, a spider plot can be employed to

414 evaluate the performance of any multifunctional entity, including performances of several

415 prediction models on a particular performance evaluation index calculated using the actual and

416 model-predicted data. In this effort, spider plots were drawn to illustrate performances of the

417 developed prediction models on two benefits (Accuracy and R) and four cost indices (RMSE,

418 MAE, MAD, MBE). The results are presented in Fig. 8 which demonstrate the superiority of the 419 HFS model over the M5 Model Tree, FIS, and RT.

\subsection{Ranking of the developed ET $T_{0}$ prediction models}

422 It is an apparent and well-established fact that prediction models behave differently in terms of 423 prediction accuracies when different performance evaluation criteria are used to compute the 
424 prediction performances (Roy et al., 2020). This contrasting behavior of prediction models needs

425 to be resolved to provide an unbiased suitability of an individual model. To resolve this

426 contradictory behavior of models, Shannon's entropy-based decision theory was applied to provide

427 ranking of the considered prediction models. This ranking approach made use of six performance

428 indices, three of them were benefit indices (the higher values designate better model accuracy: R,

429 NS, and IOA) while the remaining three were cost indices (the lower values designate better model

430 accuracy: RMSE, MAE, and MAD). These benefit and cost indices were incorporated in

431 calculating the weights for individual prediction models. The entropy weights calculated using

432 Shannon's entropy are presented in Fig. 9.

434 It is apparent from Fig. 9 that Shannon's entropy-based decision theory determined that the HFS 435 (entropy weight $=0.93)$ model had superior performance, followed by the M5 Model Tree (entropy 436 weight $=0.90)$, FIS (entropy weight $=0.77)$, and RT (entropy weight $=0.74)$ models. It can, 437 therefore, be concluded that the PSO tuned HFS model achieved higher accuracy than the other 438 tree-based prediction models considered in this effort.

\section{8. Generalization of developed models for a new unseen test dataset}

440 The HFS and other tree-based models developed at the training station (Gazipur Sadar) were

441 validated using meteorological data obtained from a test station (Ishurdi ststion). Three distinct 442 sets of data of the test station were inputted to the developed models for predicting daily ET0, 443 which were then compared with the estimated $\mathrm{ET}_{0}$ and differrnt performance evaluation indices 444 were computed using the model predicted and FAO 56 PM estimated $\mathrm{ET}_{0}$ values. The performance 445 evaluation results in terms of various statistical indices are shown in Table 5. As the results 446 indicate, the models performed equally well when compared to the results of the training station. 
447 The model performances were satisfactory concerning the computed statistical indices: the model 448 produced higher values of accuracy, NS, IOA, and R as well as lower values of RMSE, NRMSE, 449 and MBE for all three datasets.

\section{[Table 5]}

\section{9. Conclusion}

452 The potential of the PSO tuned HFS modelling approach for the prediction of $\mathrm{ET}_{0}$ using climatic 453 variables was explored in this research. The study revealed that modelling of daily $\mathrm{ET}_{0}$ can 454 efficiently be predicted using the Fuzzy logic-based HFS model, specifically when TMF is 455 employed to develop the Sugeno type FIS for the construction of HFS. Five input attributes 456 (climatic variables) such as solar radiation, relative humidity, minimum and maximum 457 temperatures, and wind speed were utilized to predict the daily $\mathrm{ET}_{0}$. The HFS was constructed 458 from five FIS objects built using the ranked input attributes (correlations of the input attributes 459 with the output attribute, $\mathrm{ET}_{0}$ ). The input-output MFs and the rule bases of the constructed HFS 460 were then tuned in two steps with PSO that provided fast convergence of the parameter tuning 461 process for the training dataset. The train and validation with the dataset of the train station 462 revealed that the developed HFS adequately mapped the input-output patterns of the train Station 463 dataset. Therefore, an HFS can effectively be applied in predicting ET $_{0}$ with climatic variables as 464 inputs. Nevertheless, it is of crucial importance to test the developed HFS model's performance 465 outside the training and validation dataset. To test the reliability of the HFS model in predicting $466 \mathrm{ET}_{0}$ for the unseen test dataset (used neither to train nor validate the developed HFS), the 467 developed HFS model was employed to predict $\mathrm{ET}_{0}$ for the test dataset. Results revealed the 468 potentiality of the HFS model in accurate and reliable prediction of $\mathrm{ET}_{0}$ for the test dataset. The 
469 proposed modelling tool provides a promising approach for $\mathrm{ET}_{0}$ estimation in sub-tropical 470 climates.

471 The study applied data from one weather station and the developed models were tested for the

472 unseen test dataset. It is worthwhile to assess the usability of the proposed HFS modelling approach

473 by including weather stations with varying climatic zones. Future research may be directed

474 towards exploring and comparing other bio-inspired optimization algorithms for the parameter 475 tuning process of the HFS models.

\section{Declarations}

477 Funding: This research did not receive any specific grant from funding agencies in the public, 478 commercial, or not-for-profit sectors.

479 Conflicts of interest/Competing interests: The authors declare that there is no conflict of interest.

480 Availability of data and material: Datasets and other materials are available with the authors, and

481 may be accessible at any time upon request.

482 Code availability: MATLAB codes are available with the first author.

\section{References}

484 Allen RG, Pereira LS, Raes D, Smith M (1998) Crop evapotranspiration— guidelines for computing crop water requirements. FAO Irrig Drain Pap No 56, Rome

Bhattacharya B, Solomatine DP (2005) Neural networks and M5 model trees in modelling water level-discharge relationship. Neurocomputing 63:381-396. https://doi.org/https://doi.org/10.1016/j.neucom.2004.04.016

489 Breiman L, Friedman JH, Olshen RA, Stone CJ (1984) Classification and regression trees. $490 \quad$ Wadsworth International, CA, USA. 
491 Chen H, Huang JJ, McBean E (2020) Partitioning of daily evapotranspiration using a modified

492

493

494

495

496

497

498

499

500

501

502

503

504

505

506

507

508

509

510

511

512 shuttleworth-wallace model, random Forest and support vector regression, for a cabbage farmland. Agric Water Manag 228:105923. https://doi.org/https://doi.org/10.1016/j.agwat.2019.105923

Chia MY, Huang YF, Koo CH (2020) Support vector machine enhanced empirical reference evapotranspirationestimation with limitedmeteorological parameters. Comput Electron Agric 175:105577. https://doi.org/https://doi.org/10.1016/j.compag.2020.105577

Ferreira LB, da Cunha FF (2020) New approach to estimate daily reference evapotranspiration based on hourly temperature and relative humidity using machine learning and deep learning. Agric Water Manag 234:106113. https://doi.org/https://doi.org/10.1016/j.agwat.2020.106113

Ferreira LB, da Cunha FF, de Oliveira RA, Fernandes Filho EI (2019) Estimation of reference evapotranspiration in Brazil with limited meteorological data using ANN and SVM - A new approach. J Hydrol 572:556-570. https://doi.org/https://doi.org/10.1016/j.jhydrol.2019.03.028

Francone FD (2001) Owner's manual: Fast genetic programming based on AIMLearning technology

Gocić M, Arab Amiri M (2021) Reference evapotranspiration prediction using neural networks and optimum time lags. Water Resour Manag 35:1913-1926. https://doi.org/10.1007/s11269-021-02820-8

Gupta HV, Sorooshian S, Yapo PO (1999) Status of automatic calibration for hydrologic models: Comparison with multilevel expert calibration. J Hydrol Eng 4:135-143. 
https://doi.org/10.1061/(ASCE)1084-0699(1999)4:2(135)

514 Han D, Cluckie ID, Karbassioun D, et al (2002) River flow modelling using fuzzy decision trees.

515 Water Resour Manag 16:431-445. https://doi.org/10.1023/A:1022251422280

516 Heinemann AB, Oort PAV, Fernandes DS, Maia A (2012) Sensitivity of APSIM/ORYZA model 517 due to estimation errors in solar radiation. Bragantia 71:572-582

518 Jang J-SR, Sun C-T, Mizutani E (1997) Neuro-fuzzy and soft computing: A computational 519

520

521

522

Jekabsons G (2016) M5PrimeLab: M5' regression tree, model tree, and tree ensemble toolbox approach to learning and machine intelligence. Prentice-Hall, Upper Saddle River, New Jersey

523

524

525

526

527

528

529

530

531

532

533

534 for Matlab/Octave

Karbasi M (2018) Forecasting of multi-step ahead reference evapotranspiration using waveletGaussian process regression model. Water Resour Manag 32:1035-1052. https://doi.org/10.1007/s11269-017-1853-9

Kennedy J, Eberhart R (1995) Particle swarm optimization. In: Proceedings of ICNN'95 International Conference on Neural Networks. pp 1942-1948 vol.4

Kisi O (2016) Modeling reference evapotranspiration using three different heuristic regression approaches. Agric Water Manag 169:162-172. https://doi.org/https://doi.org/10.1016/j.agwat.2016.02.026

Kord M, Asghari Moghaddam A (2014) Spatial analysis of Ardabil plain aquifer potable groundwater using fuzzy logic. J King Saud Univ - Sci 26:129-140. https://doi.org/https://doi.org/10.1016/j.jksus.2013.09.004

Krzywinski M, Altman N (2017) Classification and regression trees. Nat Methods 14:757-758. 
https://doi.org/10.1038/nmeth.4370

Li M-F, Tang X-P, Wu W, Liu H-B (2013) General models for estimating daily global solar radiation for different solar radiation zones in mainland China. Energy Convers Manag 70:139-148. https://doi.org/https://doi.org/10.1016/j.enconman.2013.03.004

Liu SM, Xu ZW, Zhu ZL, et al (2013) Measurements of evapotranspiration from eddycovariance systems and large aperture scintillometers in the Hai River Basin, China. J Hydrol 487:24-38. https://doi.org/https://doi.org/10.1016/j.jhydrol.2013.02.025

Martí P, González-Altozano P, López-Urrea R, et al (2015) Modeling reference evapotranspiration with calculated targets. Assessment and implications. Agric Water Manag 149:81-90. https://doi.org/https://doi.org/10.1016/j.agwat.2014.10.028

Mathworks (2021) Technical documentation. In: Fuzzy trees. https://au.mathworks.com/help/fuzzy/fuzzy-trees.html. Accessed 5 May 2021

Müller J, Piché R (2011) Mixture surrogate models based on Dempster-Shafer theory for global optimization problems. J Glob Optim 51:79-104. https://doi.org/10.1007/s10898-010-9620-

Petković B, Petković D, Kuzman B, et al (2020) Neuro-fuzzy estimation of reference crop evapotranspiration by neuro fuzzy logic based on weather conditions. Comput Electron Agric 173:105358. https://doi.org/https://doi.org/10.1016/j.compag.2020.105358

Quinlan JR (1992) Learning with continuous classes. In: Proceedings of Australian Joint Conference on Artificial Intelligence. Hobart 16-18 November, pp 343-348

Reis MM, da Silva AJ, Zullo Junior J, et al (2019) Empirical and learning machine approaches to estimating reference evapotranspiration based on temperature data. Comput Electron Agric 
558 Roy DK, Barzegar R, Quilty J, Adamowski J (2020) Using ensembles of adaptive neuro-fuzzy

Roy DK, Datta B (2019) An ensemble meta-modelling approach using the Dempster-Shafer theory of evidence for developing saltwater intrusion management strategies in coastal aquifers. Water Resour Manag 33:775-795. https://doi.org/10.1007/s11269-018-2142-y

Roy DK, Datta B (2020) Saltwater intrusion prediction in coastal aquifers utilizing a weightedaverage heterogeneous ensemble of prediction models based on Dempster-Shafer theory of evidence. Hydrol Sci J 1-13. https://doi.org/10.1080/02626667.2020.1749764

Salam R, Islam ARMT (2020) Potential of RT, bagging and RS ensemble learning algorithms for reference evapotranspiration prediction using climatic data-limited humid region in Bangladesh. J Hydrol 590:125241. https://doi.org/https://doi.org/10.1016/j.jhydrol.2020.125241

Shannon CE (1948) A mathematical theory of communication. Bell Syst Tech J 27:379-423. https://doi.org/10.1002/j.1538-7305.1948.tb01338.x

Siddique N, Adeli H (2013) Computational intelligence: Synergies of fuzzy logic, neural networks and evolutionary computing. Wiley, Hoboken, NJ

Sikorska-Senoner AE, Seibert J (2020) Flood-type trend analysis for alpine catchments. Hydrol Sci J 65:1281-1299. https://doi.org/10.1080/02626667.2020.1749761

578 Sugeno M (1985) Industrial applications of fuzzy control. Elsevier Science Inc.655 Avenue of 
580

581

582

583

584

585

586

587

588

589

590

591

592

593

594

595

596

597

598

599

600

601

Sugeno M, Yasukawa T (1993) A fuzzy-logic-based approach to qualitative modeling. IEEE Trans Fuzzy Syst 1:7. https://doi.org/10.1109/TFUZZ.1993.390281

Takagi T, Sugeno M (1985) Fuzzy identification of systems and its applications to modeling and control. IEEE Trans Syst Man Cybern SMC-15:116-132. https://doi.org/10.1109/TSMC.1985.6313399

Wang S, Lian J, Peng Y, et al (2019) Generalized reference evapotranspiration models with limited climatic data based on random forest and gene expression programming in Guangxi, China. Agric Water Manag 221:220-230. https://doi.org/https://doi.org/10.1016/j.agwat.2019.03.027

Wei C-C, Hsu N-S (2008) Derived operating rules for a reservoir operation system: Comparison of decision trees, neural decision trees and fuzzy decision trees. Water Resour Res 44:2428. https://doi.org/10.1029/2006WR005792

Zheng H, He J, Zhang Y, et al (2019) A general model for fuzzy decision tree and fuzzy random forest. Comput Intell 35:310-335. https://doi.org/https://doi.org/10.1111/coin.12195 


Variables
Entire dataset

Minimum temperature, ${ }^{\circ} \mathrm{C}$

Maximum temperature, ${ }^{\circ} \mathrm{C}$

Relative humidity, \%

Wind speed, $\mathbf{m} \mathbf{~ s}^{\mathbf{- 1}}$

Sunshine duration, $\mathrm{h}$

First half data

Minimum temperature, ${ }^{\circ} \mathrm{C}$

Maximum temperature, ${ }^{\circ} \mathrm{C}$

Relative humidity, \%

Wind speed, $\mathbf{m ~ s}^{-1}$

Sunshine duration, $\mathrm{h}$

Second half data

Minimum temperature, ${ }^{\circ} \mathrm{C}$

Maximum temperature, ${ }^{\circ} \mathrm{C}$

Relative humidity, $\%$

Wind speed, $\mathbf{m ~ s}^{\mathbf{- 1}}$

Sunshine duration, $\mathrm{h}$
21.37

31.46

78.89

1.43

5.90

21.06

31.27

80.06

1.43

5.75

21.69

31.66

77.71

1.44

6.05
5.98

4.16

12.18

0.23

3.19

6.08

4.21

11.30

0.23

3.18

5.87

4.11

12.89

0.23

3.19
$-0.73$

$-0.83$

$-0.76$

$-1.23$

0.28

0.07

1.93

0.22

$-0.41$

$-0.71$

$-0.63-0.88$

$-1.10$

2.11

0.75

$-1.32$

$-1.04$

\section{8}

609

610

611

612

613

614 


\begin{tabular}{lccccccccc}
\hline \multirow{2}{*}{$\begin{array}{c}\text { Performance } \\
\text { Indices }\end{array}$} & \multicolumn{2}{c}{ HFS } & \multicolumn{2}{c}{ FIS } & \multicolumn{3}{c}{ RT } & \multicolumn{2}{c}{ M5 Model Tree } \\
\cline { 2 - 9 } & Train & Validation & Train & Validation & Train & Validation & Train & Validation \\
\hline Accuracy & 0.996 & 0.994 & 0.996 & 0.996 & 0.998 & 0.989 & 0.999 & 0.996 \\
IOA & 0.995 & 0.993 & 0.986 & 0.985 & 0.996 & 0.985 & 0.998 & 0.993 \\
MAD, mm d & -1 & 0.071 & 0.072 & 0.117 & 0.117 & 0.056 & 0.111 & 0.045 & 0.065 \\
MAE, mm d & -1 & 0.148 & 0.149 & 0.243 & 0.246 & 0.120 & 0.237 & 0.093 & 0.150 \\
MAPRE, \% & 4.374 & 4.453 & 8.228 & 8.332 & 3.441 & 6.800 & 2.735 & 4.384 \\
MBE & -0.003 & 0.010 & 0.000 & 0.022 & 0.000 & 0.023 & 0.000 & 0.004 \\
NRMSE & 0.052 & 0.056 & 0.082 & 0.084 & 0.043 & 0.084 & 0.033 & 0.059 \\
NS & 0.979 & 0.974 & 0.947 & 0.943 & 0.986 & 0.943 & 0.991 & 0.972 \\
R & 0.989 & 0.987 & 0.973 & 0.971 & 0.993 & 0.971 & 0.996 & 0.986 \\
RMSE, mm d & -1 & 0.199 & 0.211 & 0.316 & 0.316 & 0.164 & 0.316 & 0.127 & 0.221 \\
\hline
\end{tabular}

617 *HFS = Hierarchal Fuzzy Systems, FIS = Fuzzy Inference System, RT = Regression Tree, IOA = Willmott's Index of Agreement, MAD =

618 Median Absolute Deviation, MAE = Mean Absolute Error, MAPRE = Mean Absolute Percentage Relative Error, MBE = Mean Bias Error,

619

620

621

622 Table 4 Performances of HFS and other tree-based models on the applied dataset

\begin{tabular}{|c|c|c|c|c|}
\hline Performance Indices & HFS & FIS & RT & M5 Model Tree \\
\hline Accuracy & 0.989 & 0.977 & 0.981 & 0.987 \\
\hline IOA & 0.999 & 0.999 & 0.998 & 0.999 \\
\hline $\mathrm{MAD}, \mathrm{mm} \mathrm{d}^{-1}$ & 0.068 & 0.114 & 0.116 & 0.066 \\
\hline MAE, $\mathrm{mm} \mathrm{d}^{-1}$ & 0.148 & 0.225 & 0.255 & 0.158 \\
\hline MAPRE, \% & 4.420 & 7.072 & 7.344 & 4.657 \\
\hline $\mathrm{MBE}$ & 0.029 & 0.064 & 0.050 & 0.038 \\
\hline NRMSE & 0.052 & 0.075 & 0.093 & 0.063 \\
\hline NS & 0.998 & 0.995 & 0.992 & 0.996 \\
\hline $\mathrm{R}$ & 0.987 & 0.973 & 0.958 & 0.980 \\
\hline $\mathrm{RMSE}, \mathrm{mm} \mathrm{d}^{-1}$ & 0.197 & 0.288 & 0.355 & 0.240 \\
\hline
\end{tabular}


631 Table 5 Performance of the HFS and other tree-based models using climatic data from test station (Ishurdi station)

\begin{tabular}{|c|c|c|c|c|c|c|c|c|c|c|c|c|}
\hline \multirow[t]{2}{*}{ Indices } & \multicolumn{4}{|c|}{ Entire dataset } & \multicolumn{4}{|c|}{ First half data } & \multicolumn{4}{|c|}{ Second half data } \\
\hline & HFS & FIS & RT & M5 & HFS & FIS & RT & M5 & HFS & FIS & RT & M5 \\
\hline Accuracy & 0.90 & 0.87 & 0.89 & 0.86 & 0.90 & 0.86 & 0.89 & 0.87 & 0.90 & 0.88 & 0.88 & 0.85 \\
\hline IOA & 0.94 & 0.93 & 0.83 & 0.85 & 0.95 & 0.93 & 0.84 & 0.87 & 0.93 & 0.92 & 0.81 & 0.84 \\
\hline $\mathrm{MAD}, \mathrm{mm} \mathrm{d}^{-1}$ & 0.27 & 0.27 & 0.37 & 0.41 & 0.26 & 0.29 & 0.32 & 0.38 & 0.28 & 0.26 & 0.40 & 0.43 \\
\hline MAE, $\mathrm{mm} \mathrm{d}^{-1}$ & 0.46 & 0.58 & 0.68 & 0.66 & 0.44 & 0.56 & 0.64 & 0.62 & 0.49 & 0.60 & 0.72 & 0.70 \\
\hline MAPRE, \% & 12.34 & 17.04 & 16.52 & 16.19 & 12.04 & 17.06 & 15.94 & 15.55 & 12.64 & 17.02 & 17.09 & 16.82 \\
\hline MBE & -0.38 & -0.44 & -0.53 & -0.61 & -0.37 & -0.46 & -0.50 & -0.56 & -0.35 & -0.38 & -0.50 & -0.59 \\
\hline NRMSE & 0.16 & 0.19 & 0.24 & 0.23 & 0.16 & 0.19 & 0.24 & 0.23 & 0.16 & 0.18 & 0.25 & 0.23 \\
\hline NS & 0.77 & 0.70 & 0.48 & 0.53 & 0.79 & 0.71 & 0.52 & 0.58 & 0.75 & 0.68 & 0.43 & 0.48 \\
\hline $\mathrm{R}$ & 0.93 & 0.91 & 0.82 & 0.90 & 0.94 & 0.92 & 0.83 & 0.90 & 0.92 & 0.90 & 0.80 & 0.90 \\
\hline $\mathrm{RMSE}, \mathrm{mm} \mathrm{d}^{-1}$ & 0.59 & 0.68 & 0.90 & 0.85 & 0.57 & 0.67 & 0.87 & 0.81 & 0.62 & 0.69 & 0.93 & 0.88 \\
\hline
\end{tabular}




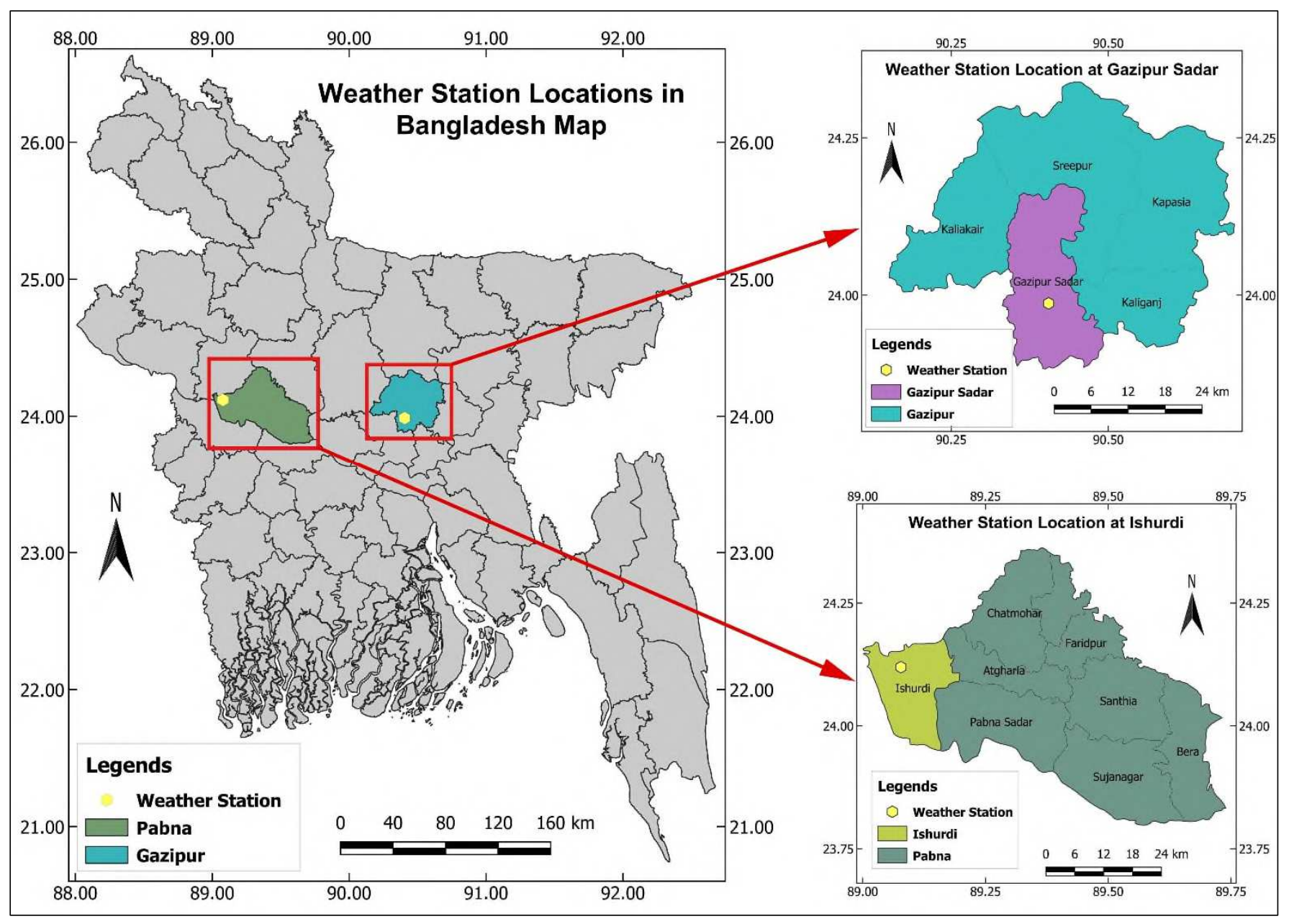

636

Fig. 1 Locations of the weather stations within the study areas

637 


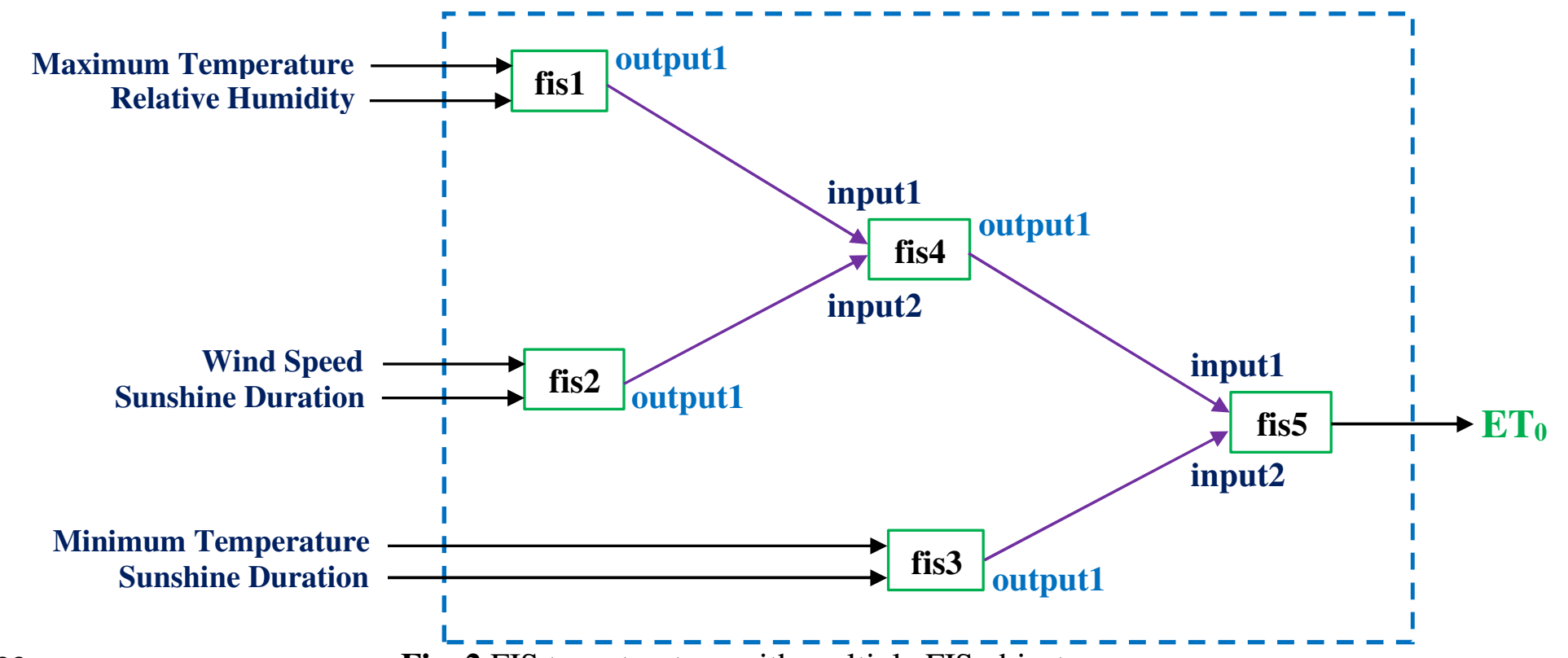

Fuzzy inference process

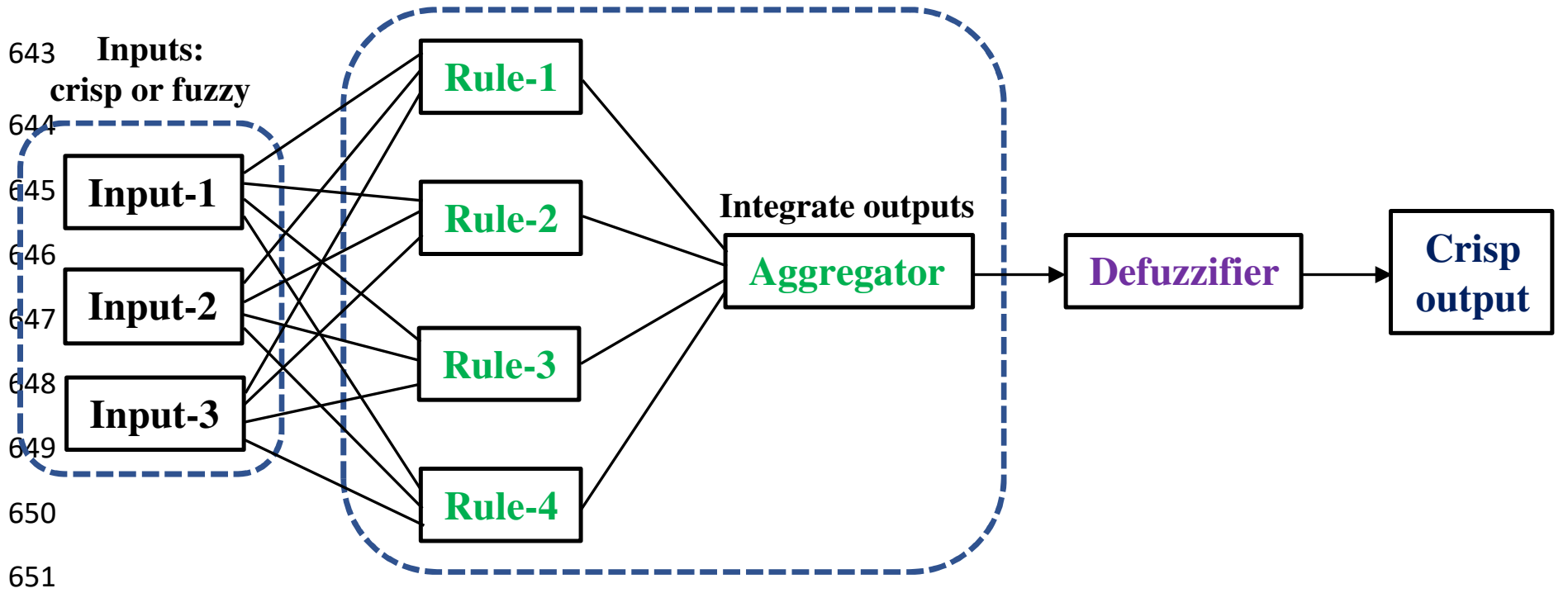

Fig. 3 Basic structure of an FIS object 
664

665

666

667

668

669

670

671

672

673

674

675

676

677

678

\section{Meteorological Variables}

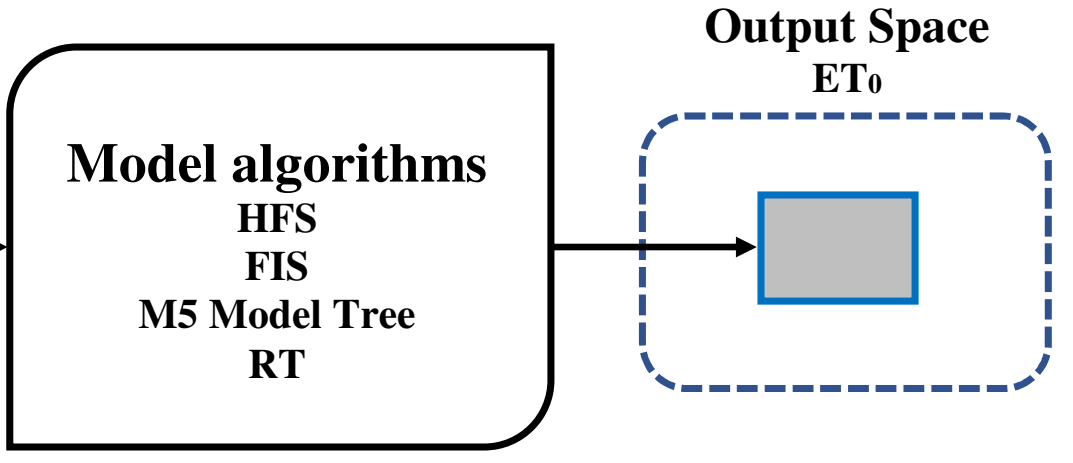

Fig. 4 Input-output mapping of prediction models for a typical $\mathrm{ET}_{0}$ modelling approach 
(a)

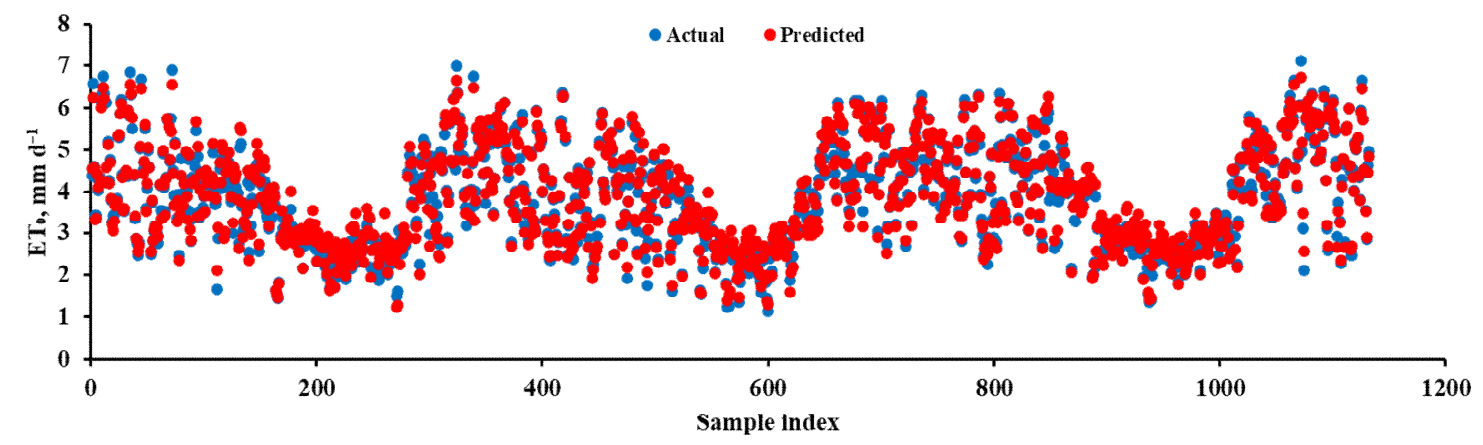

(b)

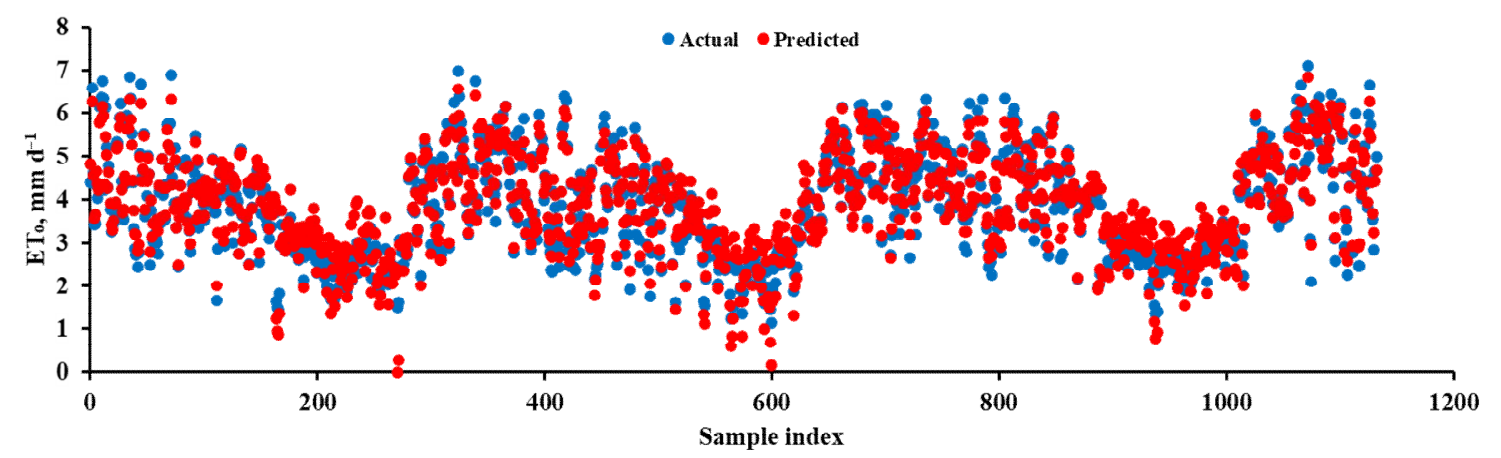

(c)

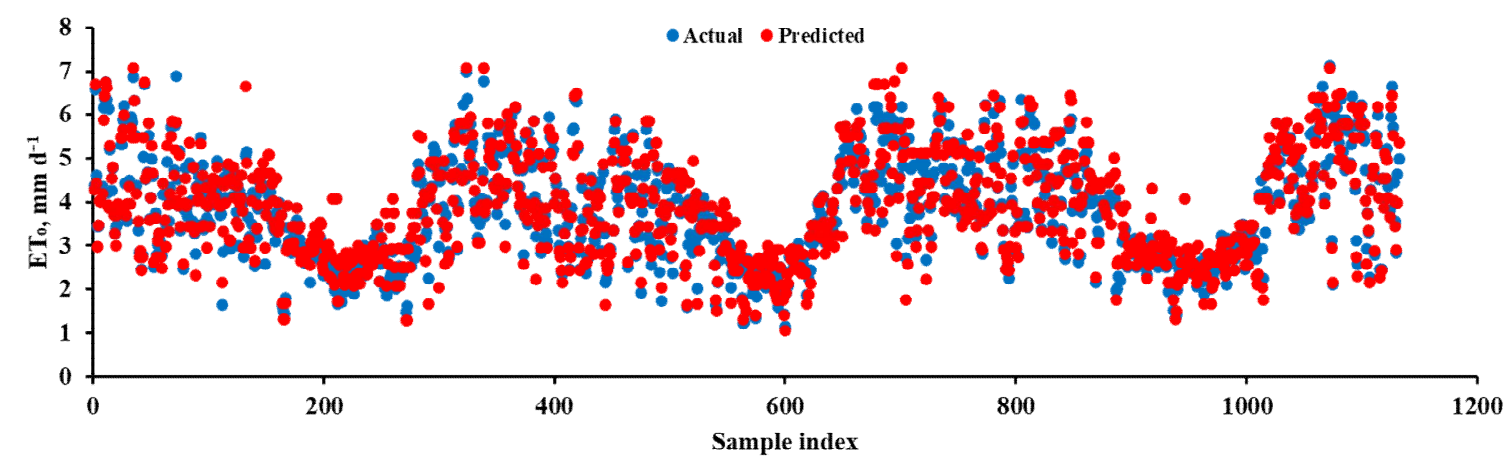

(d)

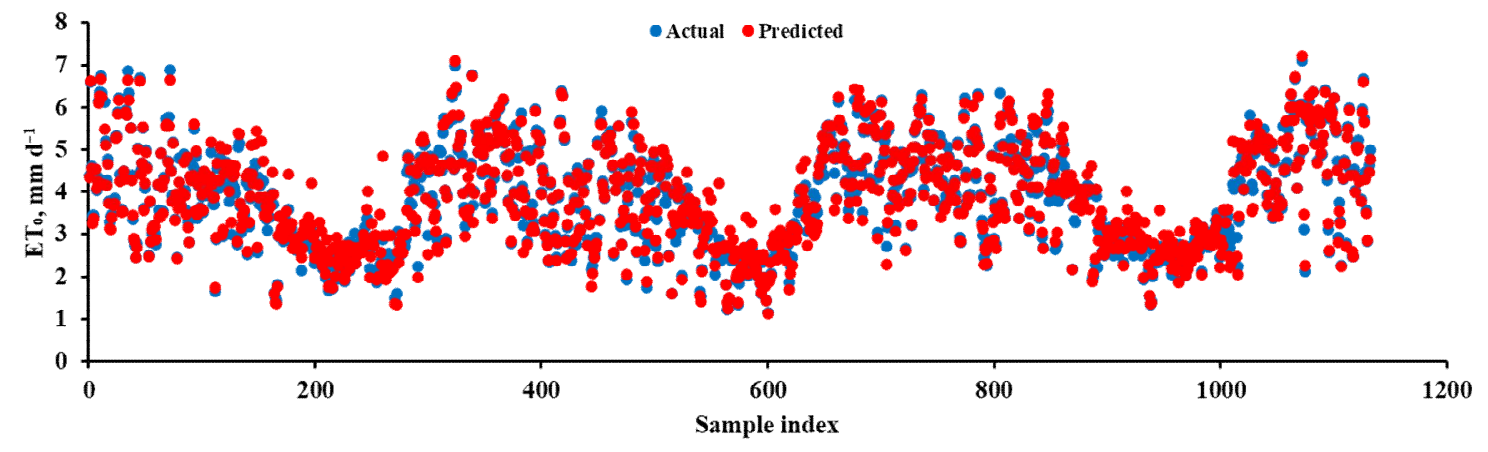

679 Fig. 5 Scatter plots of FAO 56 PM estimated and model predicted ET 0 : (a) HFS; (b) FIS; (c) RT; 680 and (d) M5 Model Tree 
Regression: $\mathbf{R}=0.98673$
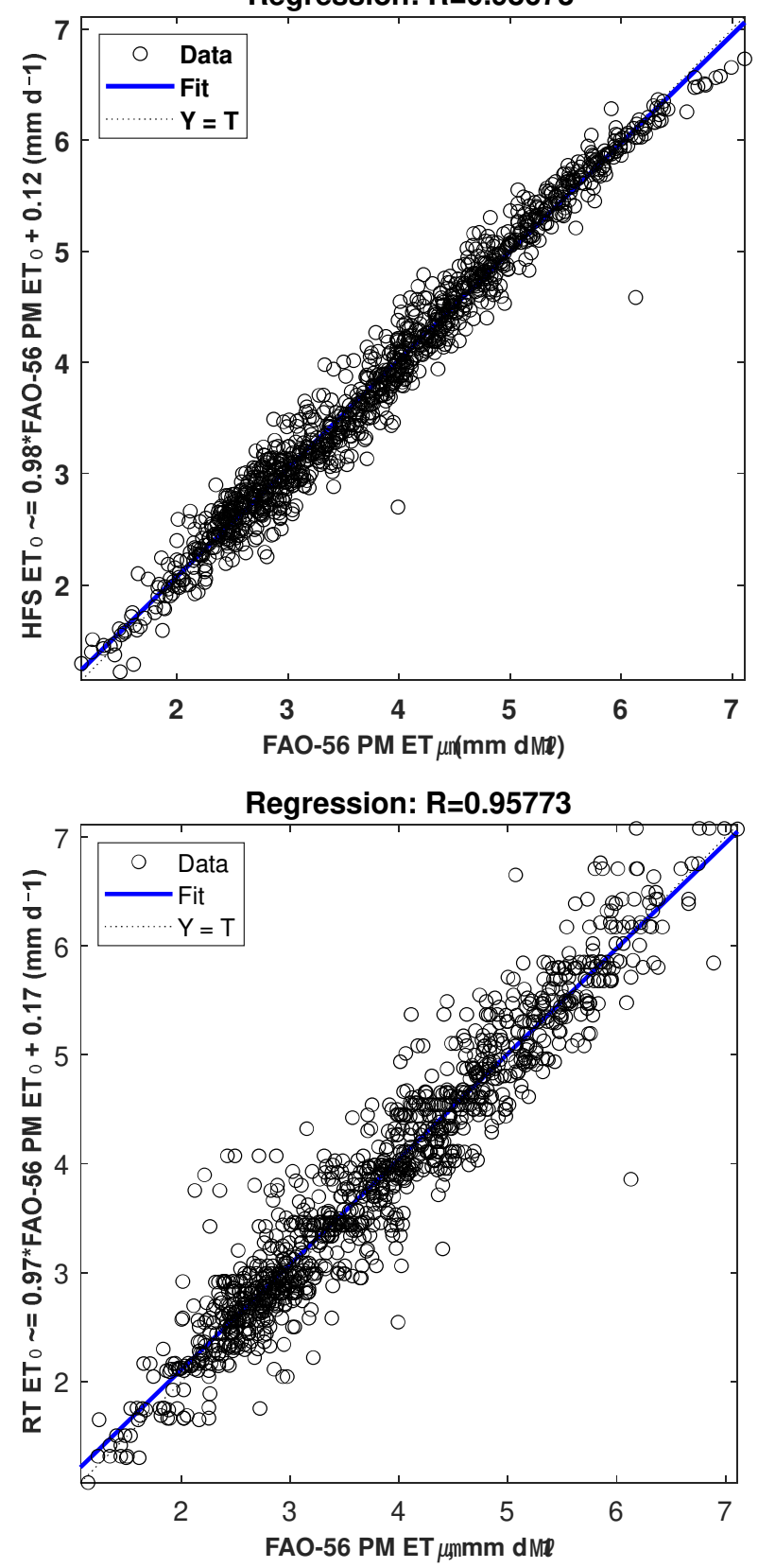

Regression: $\mathbf{R}=\mathbf{0 . 9 7 2 9 4}$

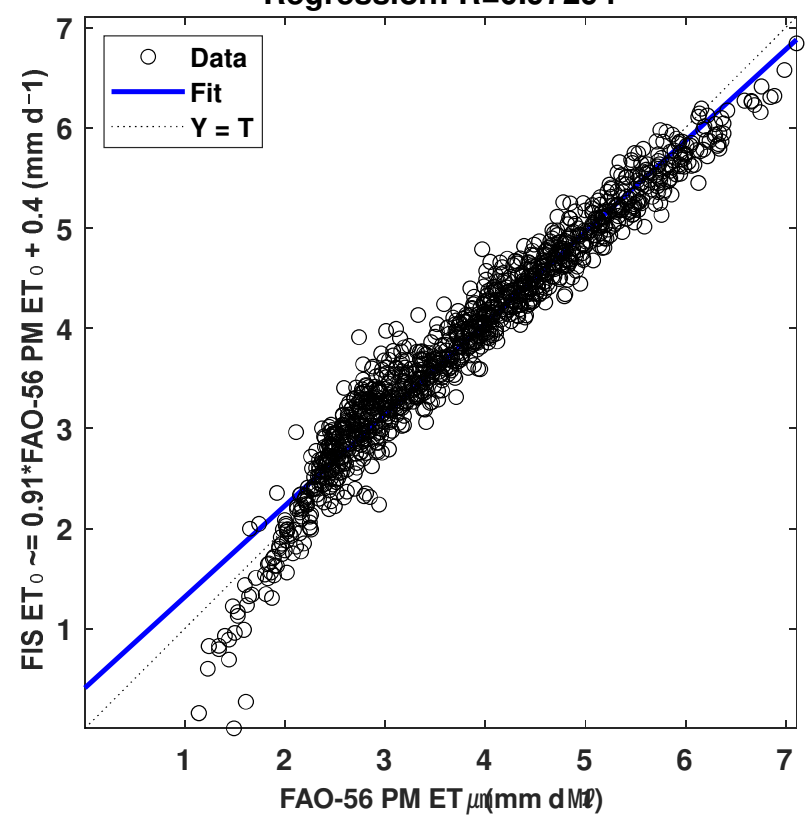

Regression: $\mathbf{R}=\mathbf{0 . 9 8 0 4 3}$

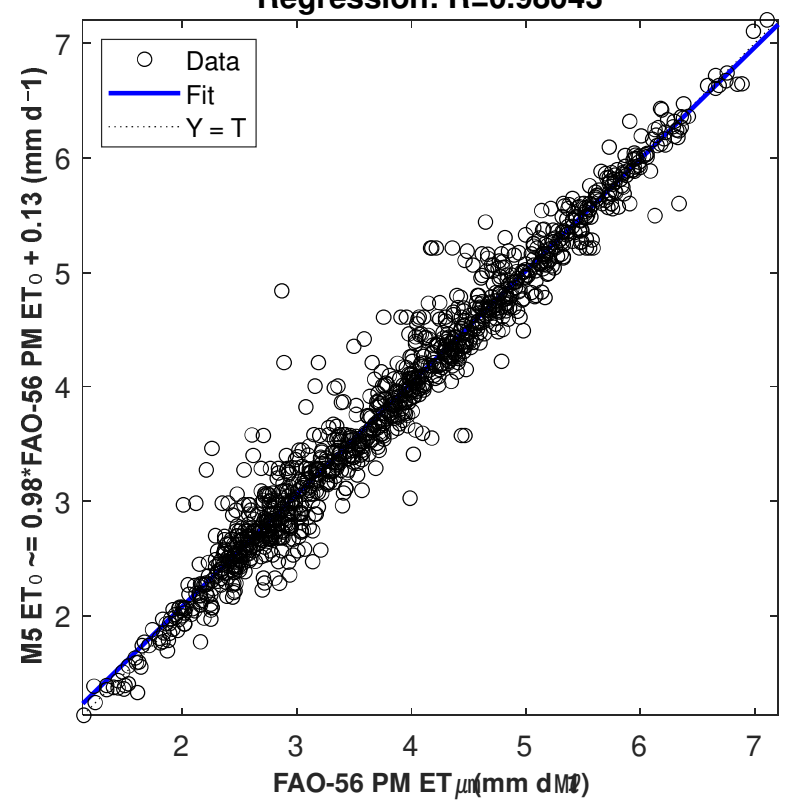

681 Fig. 6 Regression plots of the estimated (by FAO 56 PM) and predicted (by HFS, FIS, M5

682

Model Tree, and RT) $\mathrm{ET}_{0}$ during the test period 


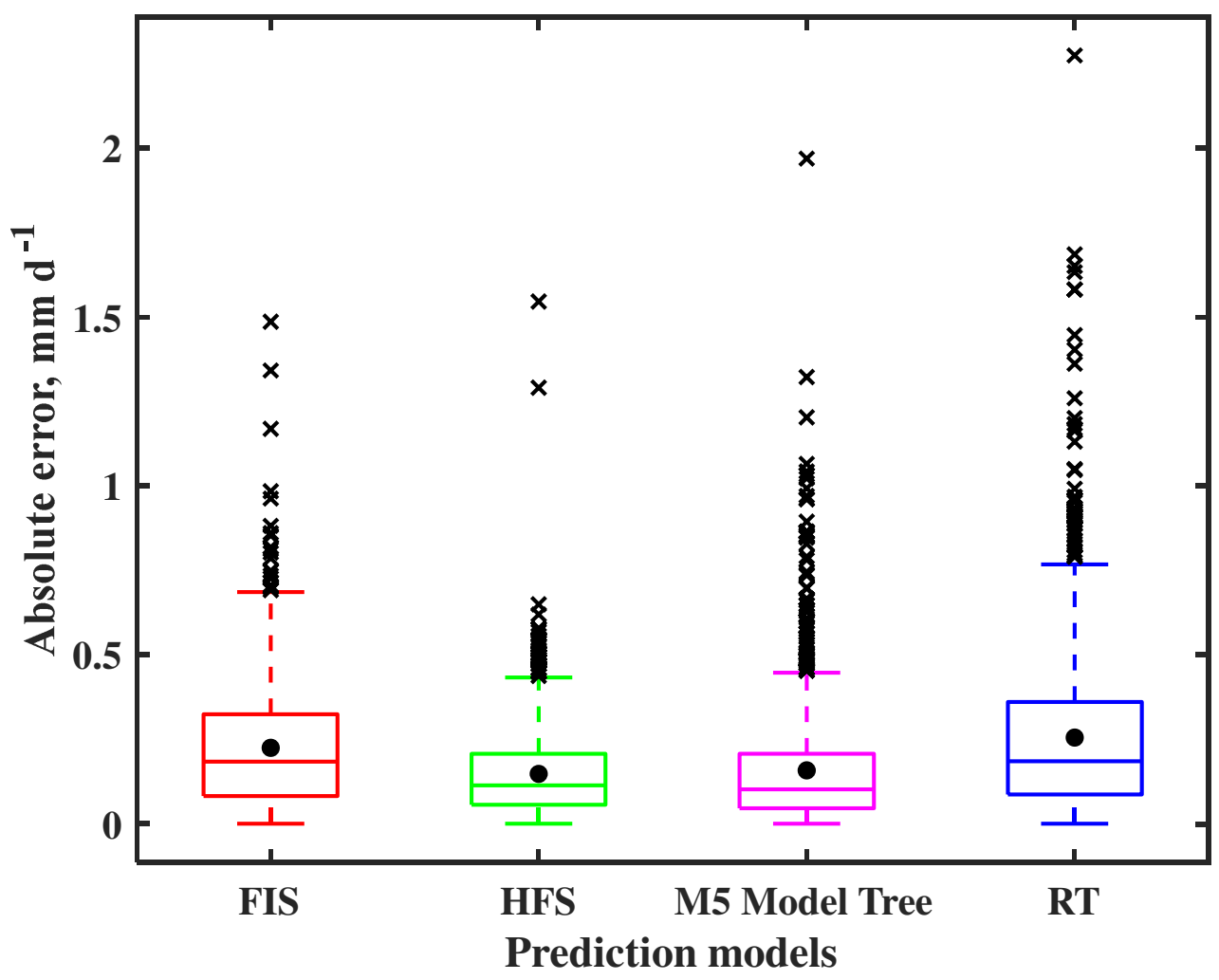

Fig. 7 Box plots of absolute errors between the FAO 56 PM estimated and model predicted daily $\mathrm{ET}_{0}$ values. The black circles represent the mean values of absolute errors. Horizontal lines inside the boxes represent the median values of absolute errors. The $\times$ symbol denotes 686 687 outliers 


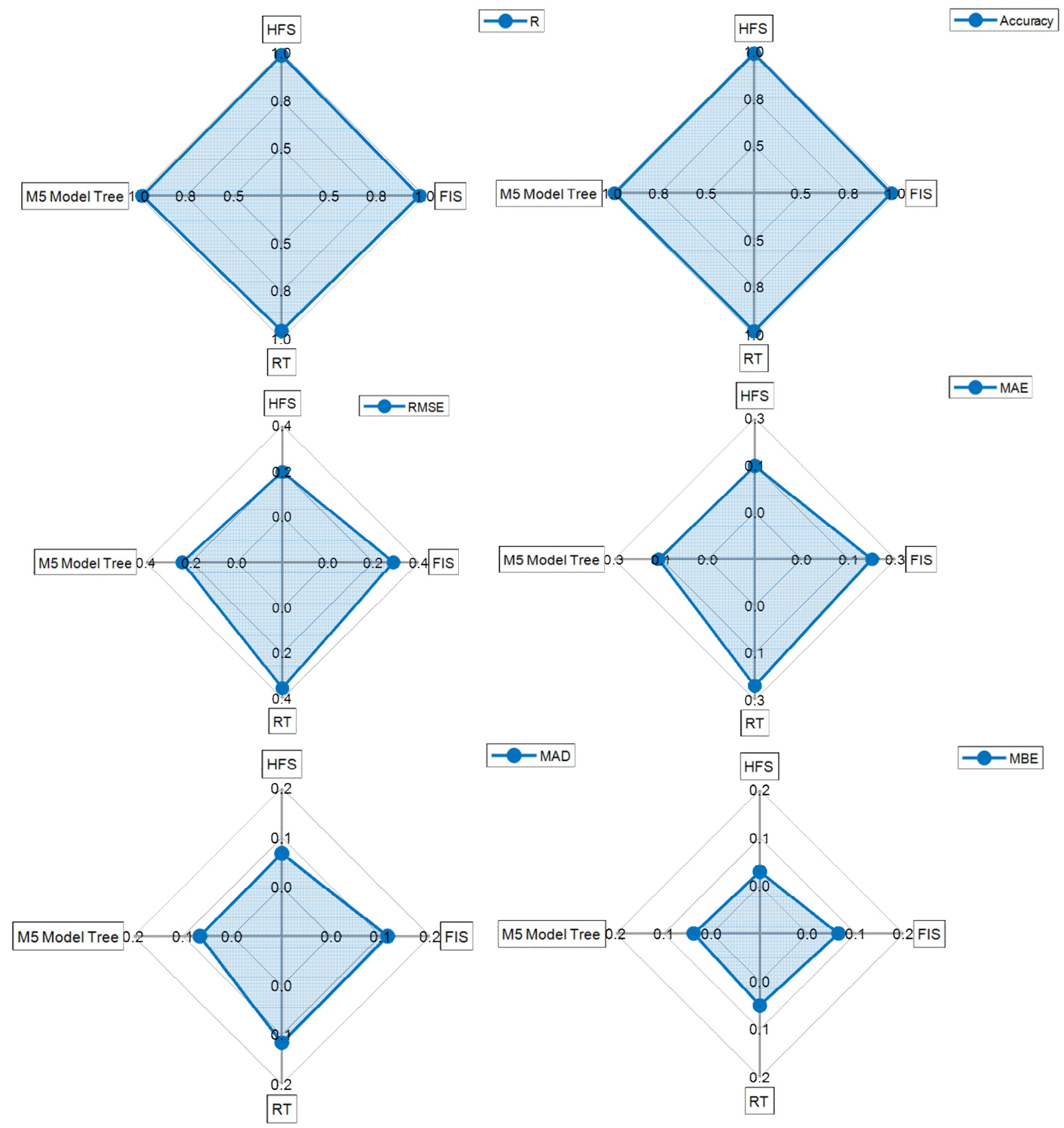

$689 \quad$ Fig. 8 Spider plots of performance evaluation indices 


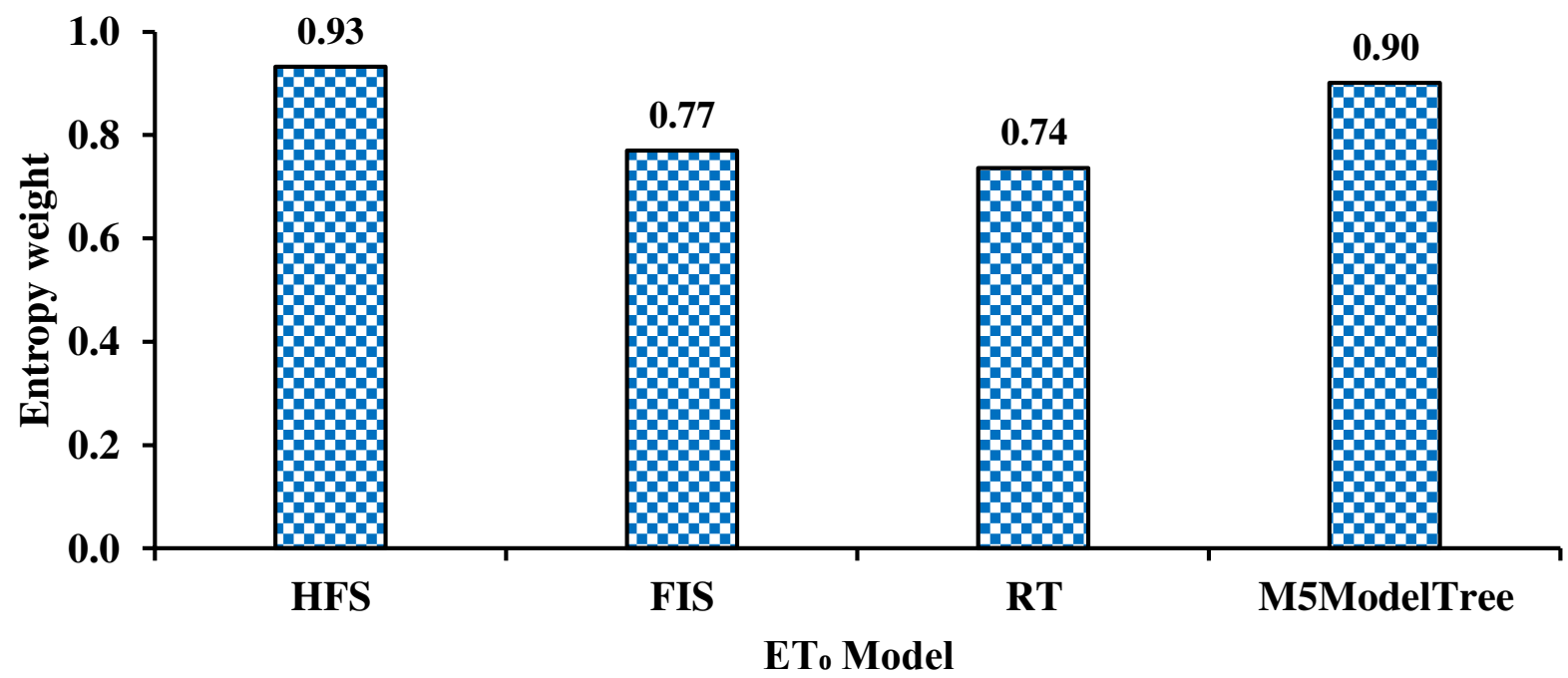

691

Fig. 9 Ranking of models based on Entropy weight

692 